\title{
Profiling structured beams using injected aerosols
}

N. Duane Loh $^{1}$, Dmitri Starodub ${ }^{1}$, Lukas Lomb ${ }^{2}$, Christina Y. Hampton ${ }^{1}$, Andrew V. Martin ${ }^{3}$ , Raymond G. Sierra ${ }^{1}$, Anton Barty ${ }^{3}$, Andrew Aquila ${ }^{4}$, Joachim Schulz 4 , Jan Steinbrener ${ }^{2}$, Robert L. Shoeman ${ }^{2}$, Stephan Kassemeyer ${ }^{2}$, Christoph Bostedt ${ }^{5}$, John Bozek ${ }^{5}$, Sascha W. Epp $^{6}$, Benjamin $\operatorname{Erk}^{6}$, Robert Hartmann ${ }^{7}$, Daniel Rolles ${ }^{6}$, Artem Rudenko ${ }^{6}$, Benedikt Rudek ${ }^{6}$, Lutz Foucar ${ }^{6}$, Nils Kimmel ${ }^{8}$, Georg Weidenspointner ${ }^{8}$, Guenter Hauser ${ }^{8}$, Peter Holl ${ }^{7}$, Emanuele Pedersoli ${ }^{9}$, Mengning Liang ${ }^{3}$, Mark S. Hunter ${ }^{10}$, Lars Gumprecht ${ }^{3}$, Nicola Coppola ${ }^{4}$ , Cornelia Wunderer ${ }^{11}$, Heinz Graafsma ${ }^{11}$, Filipe R.N.C. Maia ${ }^{12}$, Tomas Ekeberg ${ }^{13}$, Max Hantke $^{13}$, Holger Fleckenstein ${ }^{3}$, Helmut Hirsemann ${ }^{11}$, Karol Nass ${ }^{3}$, Thomas A. White ${ }^{3}$, Herbert J. Tobias ${ }^{14}$, George R. Farquar ${ }^{10}$, W. Henry Benner ${ }^{10}$, Stefan Hau-Riege ${ }^{10}$, Christian Reich $^{7}$, Andreas Hartmann ${ }^{7}$, Heike Soltau ${ }^{7}$, Stefano Marchesini ${ }^{12}$, Sasa Bajt ${ }^{11}$, Miriam Barthelmess $^{11}$, Lothar Strueder ${ }^{8}$, Joachim Ullrich ${ }^{15}$, Philip Bucksbaum ${ }^{1}$, Keith O. Hodgson ${ }^{1}$, Matthias Frank ${ }^{10}$, Ilme Schlichting ${ }^{2}$, Henry N. Chapman ${ }^{3}$ and Michael J. Bogan ${ }^{1}$.

${ }^{1}$ PULSE Institute, SLAC National Accelerator Laboratory, 2575 Sand Hill Road, Menlo Park, CA 94025 USA.

${ }^{2}$ Max-Planck-Institut für medizinische Forschung, Jahnstr. 29, 69120 Heidelberg, Germany.

${ }^{3}$ Center for Free-Electron Laser Science, DESY, Notkestraße 85, 22607 Hamburg, Germany.

4 European XFEL GmbH, Notkestraße 85, 22607 Hamburg, Germany.

${ }^{5}$ SLAC National Accelerator Laboratory, 2575 Sand Hill Road, Menlo Park, CA 94025 USA.

${ }^{6}$ Max Planck Advanced Study Group, CFEL, Notkestraße 85, 22607 Hamburg, Germany.

${ }^{7}$ PNSensor GmbH, Römerstr. 28, 80803 München, Germany.

${ }^{8}$ Max-Planck-Institut Halbleiterlabor, Otto-Hahn-Ring 6, 81739 München, Germany.

${ }^{9}$ Sincrotrone Trieste, S.S. 14-km 163,5, Trieste 34149, Italy.

${ }^{10}$ Lawrence Livermore National Laboratory, 7000 East Avenue, Livermore, CA 94551 USA.

${ }^{11}$ Photon Science, DESY, Notkestraße 85, 22607 Hamburg, Germany.

${ }^{12}$ Lawrence Berkeley National Laboratory, 1 Cyclotron Road, Berkeley, CA 94720, USA.

${ }^{13}$ Laboratory of Molecular Biophysics, Uppsala University, Husargatan 3 (Box 596), SE-751 24 Uppsala, Sweden.

${ }^{14}$ Cornell University, 244 Garden Avenue, Ithaca, NY 14853, USA.

${ }^{15}$ Max-Planck-Institut für Kernphysik, P.O. Box 1039 80, 69029 Heidelberg, Germany.

\begin{abstract}
Profiling structured beams produced by X-ray free-electron lasers (FELs) is crucial to both maximizing signal intensity for weakly scattering targets and interpreting their scattering patterns. Earlier ablative imprint studies describe how to infer the X-ray beam profile from the damage that an attenuated beam inflicts on a substrate. However, the beams in-situ profile is not directly accessible with imprint studies because the damage profile could be different from the actual beam profile. On the other hand, although a Shack-Hartmann sensor is capable of in-situ profiling, its lenses may be quickly damaged at the intense focus of hard X-ray FEL beams. We describe a new approach that probes the in-situ morphology of the intense FEL focus. By studying the translations in diffraction patterns from an ensemble of randomly injected sub-micron latex spheres, we were able to determine the non-Gaussian nature of the intense FEL beam at the Linac Coherent Light Source (SLAC
\end{abstract}

Further author information: (Send correspondence to N. Duane Loh)

N. Duane Loh: E-mail: duaneloh@slac.stanford.edu, Michael J. Bogan.: E-mail: mbogan@slac.stanford.edu

X-Ray Free-Electron Lasers: Beam Diagnostics, Beamline Instrumentation, and Applications, edited by Stefan P. Moeller, Makina Yabashi, Stefan P. Hau-Riege, Proc. of SPIE Vol. 8504, 850403 (C) 2012 SPIE · CCC code: 0277-786/12/\$18 - doi: 10.1117/12.930075 
National Laboratory) near the FEL focus. We discuss an experimental application of such a beam-profiling technique, and the limitations we need to overcome before it can be widely applied.

Keywords: aerosols, diffraction, imaging, beam profiling, beam diagnostics, Hartmann sensor, x-ray freeelectron laser, LCLS.

\section{INTRODUCTION}

The high brilliance of x-ray free-electron lasers (FELs) has enabled a new form of imaging where samples are immediately destroyed after a brief and intense exposure to pulsed radiation. ${ }^{1-5}$ With one such laser, the Linac Coherent Light Source (LCLS), ${ }^{6}$ hard x-ray pulses reaching peak powers up to $40 \mathrm{GW}$ are focused onto spots that are several microns across or smaller. Such focused pulses can produce many tens of thousands of scattered photons from single sub-micron objects. Looking forward, the goal of imaging nanometer-size particles will certainly benefit from more efficient use of the photon output from x-ray lasers beyond what optical modeling can accomplish. This may require active feedback between pulse profiling diagnostics and fine adjustments in upstream optics. For example, collecting statistics on the intensity distribution and phase curvature of focused pulses can help determine where samples should be injected to maximize their diffraction signal, and how to interpret them.

\section{METHODS FOR PULSE PROFILING}

A range of instruments are available to profile intense, hard x-ray FEL pulses. These include ablation imprints, ${ }^{7-9}$ scintillation crystals or x-ray sensitive Hartmann sensors. ${ }^{10-12}$ However, high peak intensities of FEL pulses near their small foci have made this extremely challenging, either because of the potential damage to such instruments or the difficulty in interpreting their measurements or both.

\subsection{In-focus techniques}

One could profile focused FEL pulses from the damage it inflicts on substrates once the local irradiation intensity surpasses their thermal ablation threshold. ${ }^{7}$ Threshold intensity contour of focused but attenuated single, hard x-ray pulses were measured from the damage craters they created on poly methyl methacrylate (PMMA). ${ }^{8}$ Retrieving a pulse's full intensity profile from the ablation depth in the interior of these craters require that the absorbed pulse energy be strongly localized, a condition which is more easily met for soft $\mathrm{x}$-ray ${ }^{7}$ than hard x-ray FEL pulses. ${ }^{8}$ PMMA also leads to beam profile truncation below the ablative threshold. The damage profile imprinted on silicon nitride membranes by polystyrene spheres acting as nano-lenses can help determine the near-field diffraction pattern that the latter creates when illuminated by intense soft x-ray FEL pulses. ${ }^{9}$

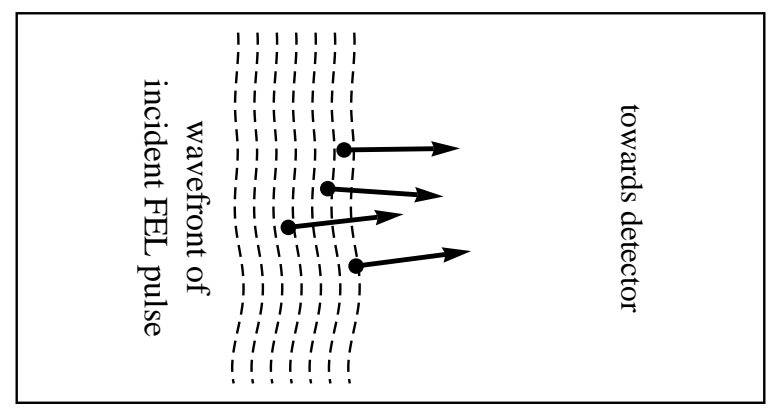

Figure 1. One dimensional phase tilts (wavefront shape) of an FEL pulse (dashed lines) cause the centers of diffraction patterns to diverge. The disks represent polystyrene spheres that are randomly injected into the page to intercept the FEL wavefront. The centers of resultant diffraction patterns travel along directions perpendicular to the beam's wavefront towards the detector, as indicated by the arrows. By selecting for single-sphere diffraction patterns, we probed each pulse's wavefront at only one such hypothetical disk each time. Generalization to two dimensional phase tilts is straightforward. 


\subsection{Out-of-focus techniques}

Out-of-focus pulse profiling techniques also exist, such as inference from the scintillation profile created by single defocused pulses on a Cerium-doped Yttrium-Aluminium-Garnet (YAG:Ce) crystal. However, the intensity of a defocused pulse must be attenuated several orders of magnitude to limit the crystal damage. Even then, irradiance-dependent non-linearities ${ }^{13}$ may distort the scintillation profile from the desired beam profile. Such non-linearities are known to cause the scintillation photon yield to change with different incident photon wavelengths ${ }^{13 *}$. Hence, absolute intensity measurements will require robust calibration across different photon energies.

Alternatively, a Hartmann sensor measures both the phase and intensity profile of individual pulses using an array of lenses that separately focuses local regions of the pulse's defocused wavefront onto distinct spots captured on a detector. This is typically done on the defocused beam to increase the sampling density of its wavefront. When the phase and intensity distribution of this defocused beam is sufficiently sampled, it can be propagated back to the focal plane of interest. The local phase tilts (wavefront shape) and intensities sampled by these lenses can be inferred from the offsets and brightness of these focal spots respectively. This has been applied to attenuated discharge-driven soft x-rays, ${ }^{10}$ hard $\mathrm{x}$-rays ${ }^{12}$ and attenuated single FEL pulses in the extreme ultraviolet and soft x-ray range. ${ }^{11}$

We note that other potential pulse profiling techniques using phase-retrieval and phase-diversity could also be explored. ${ }^{14}$

\subsection{Probing pulse focus with random aerosol spheres}

A precise morphology of the profiling instrument is often necessary if the unknown features of the pulse is to be recovered. The diffraction patterns from randomly injected, well-calibrated polystyrene spheres can be sensitive to the shape of an FEL pulse's wavefront and its intensity profile even at peak intensities of $10^{21} \mathrm{~W} / \mathrm{m}^{2}(1.5$ $\mathrm{kJ} / \mathrm{cm}^{2}$ per pulse for the most intense pulses in this paper). The working principle is analogous to a Hartmann sensor with spheres acting as randomly positioned, disposable lenses. Each injected sphere probes a local region of the FEL pulse that it intercepts: offsets in the center of the resultant diffraction pattern indicate the pulse's local wavefront shape or, equivalently, phase tilt (Fig. 1); the brightness of the pattern indicate the pulse's local intensity. These measurements are averaged over the finite depth of the pulse stream's focus, equivalent to the spot diameter of the spray of spheres (open circle in Fig. 2). Although obtaining the complete profile of individual pulses is impossible with single spheres, the character of the average FEL pulse can be reconstructed when many single sphere measurements are combined.

\section{EXPERIMENTAL PARAMETERS}

The diffraction imaging experiment for pulse profiling was performed at the Atomic Molecular and Optical Science beamline ${ }^{15}$ at the LCLS. X-ray pulses were produced at $60 \mathrm{~Hz}$ by the FEL with an average pulse energy of $2.7 \mathrm{~mJ}$ ( $4.8 \%$ pulse-to-pulse r.m.s. variation) while spanning a FWHM pulse duration of approximately 150 fs (measured from the lasing electron bunch). These pulses contained $1.2 \mathrm{keV}$ photons (wavelength $1.0 \mathrm{~nm}$, with $0.28 \%$ r.m.s. pulse-to-pulse variation in its average) thus providing a nominal $1.4 \times 10^{13}$ photons per pulse ${ }^{\dagger}$, focused using Kirkpatrick-Baez mirrors to a $10 \mu \mathrm{m}^{2}$ spot. Polystyrene spheres (70 nm radius) in solution were nebulized and injected into the FEL focus with a differentially-pumped aerodynamic lens stack. ${ }^{2,16,17}$ These spheres were randomly irradiated by FEL pulses in a narrow range of defocus planes spanning only $4.4 \%$ of the FEL pulse's Rayleigh length (Fig. 2). Diffraction patterns were recorded by a $1024 \times 1024$-pixel pnCCD x-ray detector ${ }^{18}$ comprising $75 \times 75 \mu \mathrm{m}^{2}$ pixels (Fig. 3). The detector readout was recorded at $60 \mathrm{~Hz}$, with one pattern per pulse whether or not it encountered an injected particle. From these, a software-implemented intensity filter selected background-subtracted patterns that contained scattering signal (hits), which include single and

\footnotetext{
${ }^{*}$ There would also be photon fluence effects if the ionization density created by intense x-ray pulses greatly exceed the density of luminescent centers.

${ }^{\dagger}$ The actual number of photons delivered to the FEL-sample interaction region was only a fraction of this due to optical losses upstream of the focus.
} 
multiple coincident spheres ${ }^{\ddagger}$. More than 2000 hits were collected, with 625 patterns from single spheres that did not saturate the dynamic range of the detector. Radiation damage to the latex spheres during illumination by these ultrashort pulses was undetectable at low resolution.

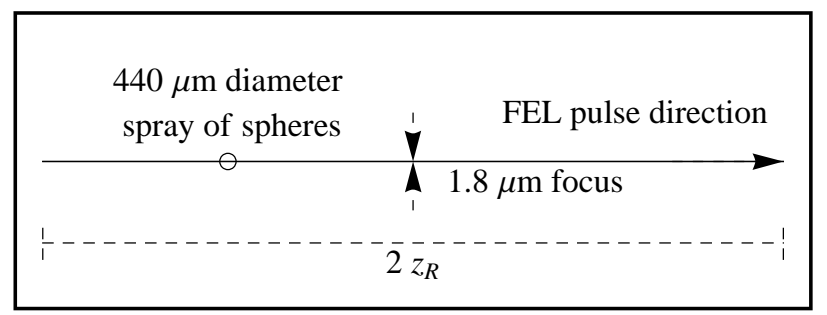

Figure 2. Sample-pulse interaction region. The image is centered on the nominal FEL pulse focus (1.8 $\mu \mathrm{m}$ pulse waist) and spans twice the pulse's Rayleigh length $z_{R}=10 \mathrm{~mm}$. FEL pulses travel along the solid horizontal line towards the right; the empty circle represents the random spray of polystyrene spheres (injected into the page). The intersection of this spray and the line of pulses define the interaction region, whose location is undetermined but expected to be within $z_{R}$ of the pulse focus along the line of pulses.

\section{DIFFRACTION DATA}

\subsection{Centering diffraction data}

The local phase tilt (wavefront shape) on a pulse that each sphere randomly intercepts caused the resultant diffraction pattern to translate (one-dimensional illustration in Fig. 1). Each pattern was centered by identifying the pixel about which the pattern was most azimuthally symmetric. Presumably, a two-dimensional sphere diffraction pattern will be most compatible with its angular average when the pattern is properly centered. Using this as a heuristic, azimuthally averaged intensities $I\left(q_{c}\right)$ around each candidate center $c$ were computed then scored against the original two-dimensional pattern assuming such a center $I\left(\mathbf{q}_{c}\right)$ :

$$
\operatorname{argmax}_{c}\left[\sum_{\left|\mathbf{q}_{c}\right|=q_{c}} \log \left(I\left(q_{c}\right)\right) \log \left(I\left(\mathbf{q}_{c}\right)\right)\right] .
$$

The candidate center with the highest score was taken to be the correct central pixel. The logarithm of the diffraction intensities were used to increase the sensitivity of this scoring over a wider dynamic range of intensities. This scoring was done for intensities at small diffraction angles, where each sphere's high-resolution deviation from sphericity is least manifest, while constraining the gap between the two detector halves to be 21 pixels (as estimated from maximizing the fringe visibility in the radial average of the brightest, centered sphere diffraction patterns). An exhaustive search for candidate centers $c$ was restricted to pixels in a $17 \times 17$-pixel region centered on the nominal center. No patterns had centers beyond a $11 \times 11$-pixel region.

\subsection{Fitting sphere radii and incident intensities}

Centered patterns of single spheres were expected to fit the scalar diffraction intensities ${ }^{\S}$

$$
I_{\mathrm{sph}}(q, r)=I_{0}\left[\frac{(\sin (q r)-q r \cos (q r))}{(q r)^{3}}\right]^{2}
$$

where the magnitude of the spatial frequency $\mathbf{q}$ is denoted as $q$, radius of a polystyrene sphere as $r$, and the fit parameter $I_{0}$ is related to the forward scattering cross section and incident photon fluence. We found both $r$ and

\footnotetext{
${ }^{\ddagger}$ The resultant hit rate was $1.6 \mathrm{~Hz}$.

$\S$ We only fit to elastic scattering. As such, Born approximation is valid in our case, and there is negligible 'Ewald sphere liftoff' given the spheres' size and detector geometry. The spheres are also much larger than the photon wavelength, making Mie theory fits unnecessary.
} 

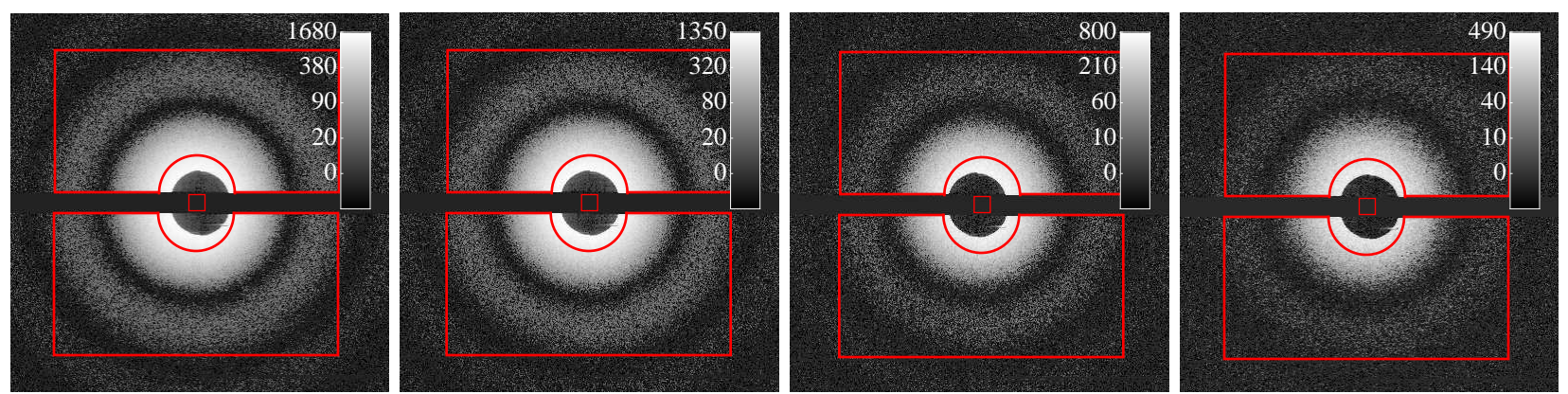

Figure 3. Four centered diffraction patterns from different single polystyrene spheres. Each figure shows the logarithm of the detector counts in the $400 \times 400$-pixel central portion of each pattern (counts in grayscale bar). The horizontal band in the middle of each diffraction patterns is the gap between the two detector panels through which unscattered photons pass; two darkened quasi-semicircular regions on the detector did not have pixels for similar reasons. Candidate centers of each pattern were restricted to the $17 \times 17$-pixel box in the middle of the pattern, selecting for the center about which the intensities in the two 'u-shape' regions (outlined in red) appear most azimuthally-symmetric. In other words, the original diffraction pattern is translated to maximize the azimuthal symmetry of the pattern in these ' $u$-shape' pixel regions, which are fixed in the centered pattern. This causes the darkened quasi-semicircular pixel regions to move about with respect to these ' $u$-shape' regions.

$I_{0}$ for each pattern, which also gives us the incident photon intensity $I_{\text {inc }}$ on each sphere. The detector readout of a single sphere diffraction pattern at spatial frequency $q=0$ is expected to be:

$$
I_{\mathrm{sph}}(0, r)=I_{\mathrm{inc}} G_{\mathrm{det}} Q_{\mathrm{eff}} r_{e}^{2} \Delta \Omega\left(\bar{f} N_{\mathrm{A}} \frac{\rho_{\mathrm{PSL}} 4 \pi r^{3}}{3 M_{\mathrm{PSL}}}\right)^{2},
$$

where the density of polystyrene $\rho_{\mathrm{PSL}}$, provided by the manufacturer, is $1.05 \mathrm{~g} / \mathrm{cm}^{3} ; N_{\mathrm{A}}$ is Avogadro's constant; $M_{\mathrm{PSL}}$ is the molar weight of polystyrene monomers $\left(\mathrm{C}_{8} \mathrm{H}_{8}\right), 104 \mathrm{~g} / \mathrm{mol} ; \bar{f}$ is the average scattering factor of polystyrene monomers for $1.2 \mathrm{keV}$ radiation, $58.3 ; r_{e}$ is the classical electron radius, $2.8 \times 10^{-15} \mathrm{~m} ; \Delta \Omega$ is the solid angle subtended by each detector pixel, $1.1 \times 10^{-8}$ steradians; $G_{\text {det }}$ is the detector's gain, 7 counts per photon; and $Q_{\text {eff }}$ is its quantum efficiency for $1.2 \mathrm{keV}$ photons, 0.9 efficiency.
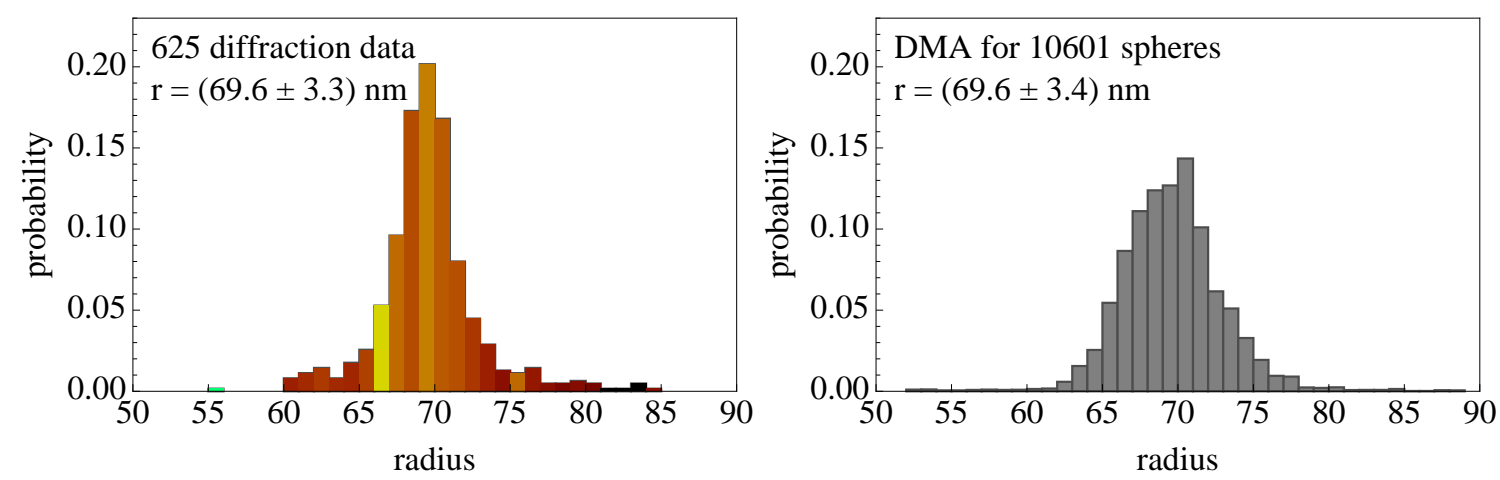

Figure 4. Calibrating the distribution of sphere radii $r$. Left: centered diffraction patterns of 625 single polystyrene spheres were fitted to the scalar diffraction model (2) to obtain a radius for each sphere. The histogram of radii is colored according to the average incident fluence for all the spheres that were sized within each histogram bin (colorbar in Fig. 5). Right: histogram of radii obtained on similar spheres using differential mobility analysis.

We calibrated the average radius of our polystyrene spheres against a separate differential mobility analyzer (DMA) measurement of polystyrene spheres similar to those used for our diffraction data. A scanning mobility particle spectrometry (TSI model 3936) was used to characterize the sphere radii distribution. An electrospray aerosol generator (TSI model 3480) aerosolized solutions of $1.5 \times 10^{12}$ to $1.5 \times 10^{13}$ polystyrene-spheres $/ \mathrm{ml}$ prepared in $25 \mathrm{mM}$ ammonium acetate in water. These electrospray droplets were then introduced into a flow 
of $1.5 \mathrm{lpm}$ air and $0.1 \mathrm{lpm} \mathrm{CO}_{2}$ to minimize corona discharge and immediately charge-reduced by exposure to ionized air created using a ${ }^{210} \mathrm{Po} \alpha$-source, then allowed to evaporate to dryness, resulting in an aerosol of discrete aerosolized spheres with a known charge distribution. The charge carried by the spheres was predominantly zero, while a small fraction was singly-charged (positively or negatively), and an even smaller fraction was doubly-charged. The size-polydisperse spheres passed into a differential mobility analyzer (DMA) (TSI, model 3081) via a conductive silicone tube and were size-classified based on their electrical mobility. A portion of the size-selected aerosol was sampled into a condensation particle counter (CPC) (TSI, model 3786) to monitor the gas phase particle concentration, typically 104 particles $/ \mathrm{cm}^{3}$ at the peak of the size distribution.

The radii measurement from DMA was considerably larger than that nominally specified by the manufacturer, PostNova Analytics (Germany), who reported a radius of $68.5 \mathrm{~nm}$ spheres and a coefficient of variance of $2.3 \%$. Our DMA measurement of the radii, $69.6 \pm 3.4 \mathrm{~nm}$, was used for calibration because of its recency and inclusion of possible post-manufacture growth factors.

Fluctuations in the photon wavelength from pulse to pulse $(0.5 \%$ r.m.s. variation) were accounted for when fitting each diffraction pattern, as were fluctuations in the total pulse energies $(2 \%$ r.m.s. variation) measured using the ultraviolet fluorescence generated from calibrated nitrogen gas detectors upstream of the interaction region. ${ }^{6}$ The incident intensities $I_{\text {inc }}$ from fitting Eq. (2) were normalized using this fluorescence readout such that all pulses had the same total energy equal to the highest measured pulse energy. Diffraction patterns whose intensities saturated the x-ray detector's dynamic range were not considered in these analyses. This is equivalent to applying an intensity lowpass filter to our data. Although we did not correct for the detector's potential non-linear response at higher incident intensities, data with poor fits to Eq. 2 were rejected

Each sphere's position in the FEL-sample interaction region were unmeasured, hence unaccounted for, but introduced less than $0.1 \%$ uncertainty in each sphere's determined radius. Since both pattern centering and sphere sizing depended primarily on the low-resolution features of the spheres, we did not attempt to correct for errors arising from higher resolution non-sphericities in our latex spheres.

\section{INTERPRETING THE RESULTS}
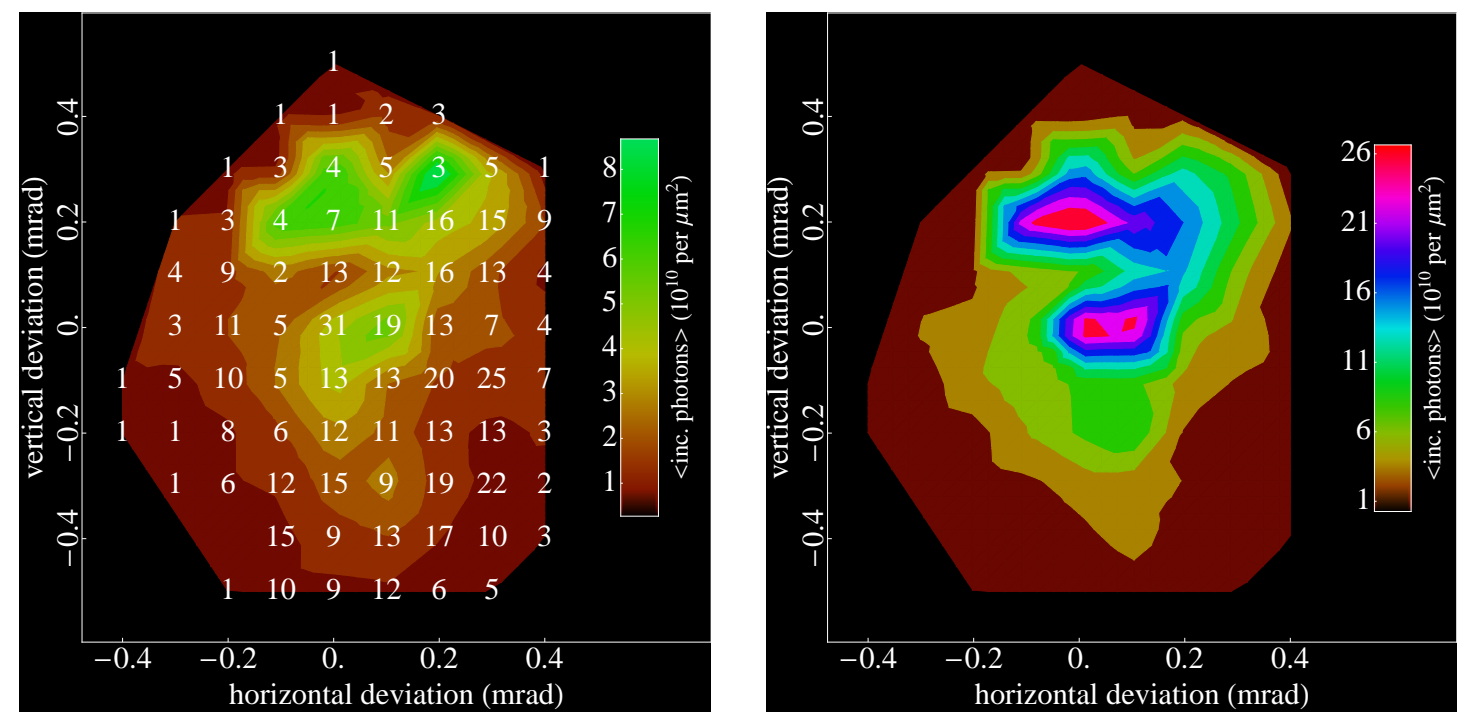

Figure 5. Translations and local incident fluence of 625 sphere diffraction patterns. Left: interpolated contour plot of the average incident photon fluence for patterns that suffer the same translation or angular deviation. The contour plot is superimposed with the number of patterns with each translation. The zero deviation position was arbitrarily chosen as the position with most patterns. Right: a similar contour plot showing the maximum local fluence.

\footnotetext{
${ }^{\top}$ Fewer than $5 \%$ of the data showed such poor fits, some of which were suspected to arise from sphere dimers where one sphere has mostly occluded another. These data were not included in the 625 patterns shown in this paper.
} 


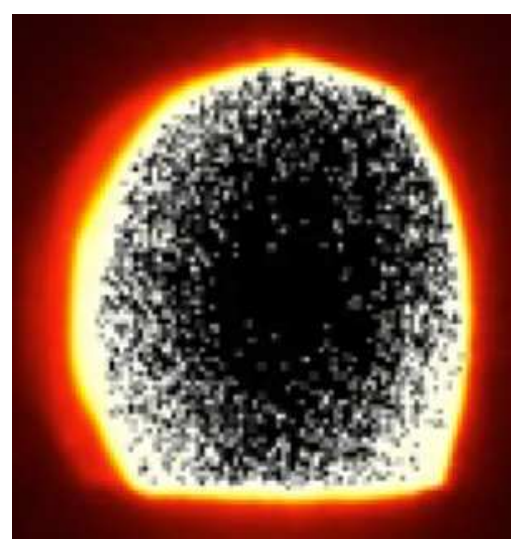

Figure 6. Outline of many attenuated FEL pulses captured on a Cerium-doped Yttrium-Aluminium-Garnet (YAG:Ce) scintillation crystal upstream of the FEL-sample interaction region. The black pixels in the middle of the pulse shape are artifacts from saturating the crystal's scintillation response.

The measured translations necessary for pattern-centering and incident fluence from fits to sphere diffraction patterns are combined in Fig. 5, where pattern translations at the detector were converted to angular deviations from the nominal center of the pulse focus. The abrupt bottom and right edges of the contour plot in Fig. 5 were from pulse truncation by the beam guards on the Kirkpatrick-Baez focusing mirrors. The numbers superimposed on Fig. 5 indicate the number of patterns that suffered each deviation. These numbers sample the distribution of phase tilts on the average FEL pulse over a $440 \mu \mathrm{m}$ depth of focus. Similar pulse outlines are also present in the scintillation figure captured on the YAG screen in Fig. 6. However, the reader should defer detailed comparisons between Figs. 5 and 6 until a unsaturated scintillation figure is recorded in future experiments.

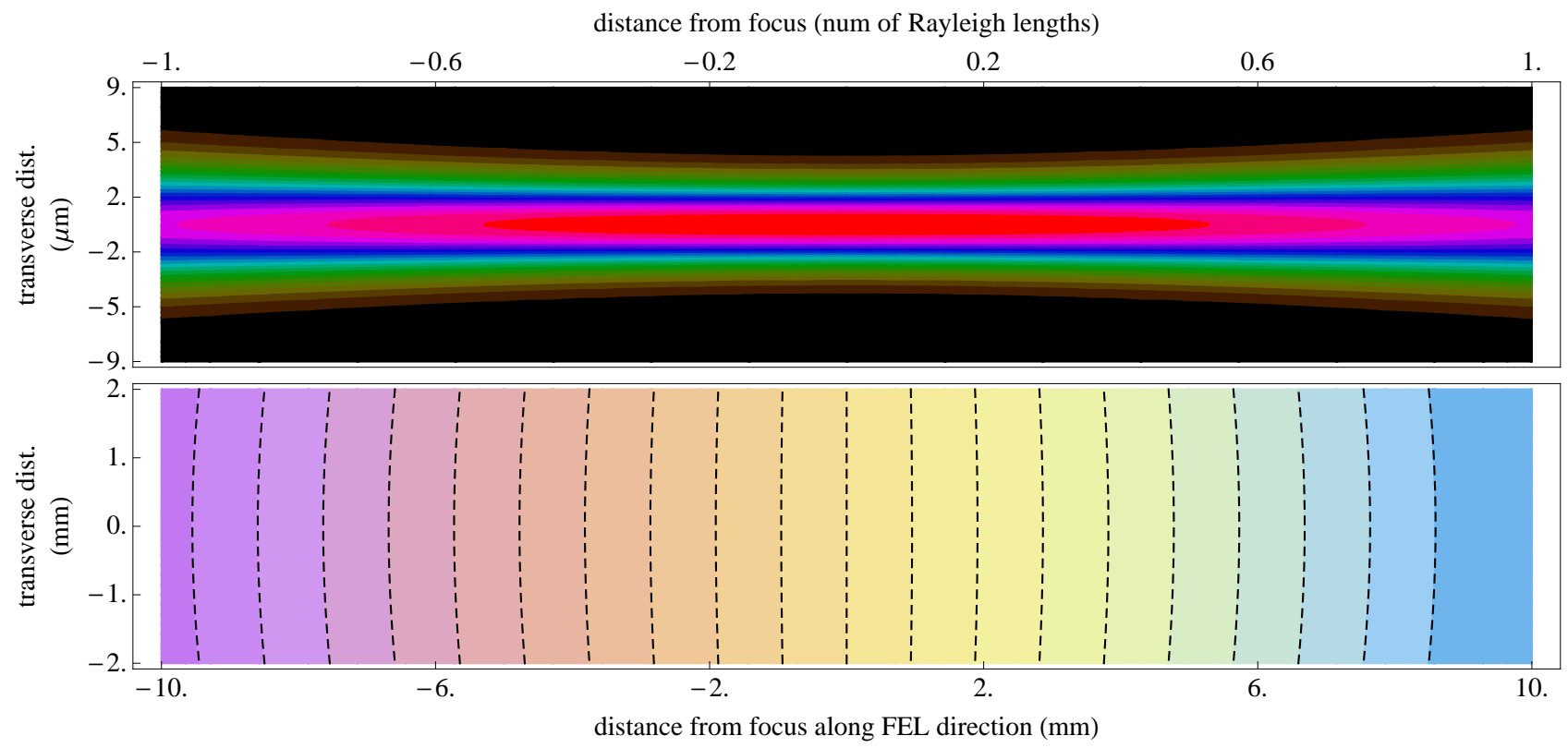

Figure 7. Intensity and phase profile of a spherical Gaussian beam (1.8 $\mu \mathrm{m}$ beam waist radius, photon wavelength 1.0 $\mathrm{nm})$. Top: intensity profile as a function of longitudinal and transverse distance. The colors indicate the contours of maximum beam intensity (red, high; green, low). Bottom: the phase curvature (dashed curves) remains strictly convex at all defocus distances and vanishes at the beam focus. The spot diameter for the spray of aerosol spheres (see Fig. 2 ) is $440 \mu \mathrm{m}$. The colors indicate the equal phase regions of the wavefront. Far from the focus, this phase curvature approaches that of a spherical wave. Note the difference in the transverse units from the top and bottom plots. These plots are azimuthally symmetric when rotated about the pulse propagation axis. 
The r.m.s. variation in divergence angle of the sphere patterns used for Fig. 5 is $0.5 \mathrm{mrad}$, which is comparable to the focused pulse's estimated divergence angle of $0.7 \mathrm{mrad}$. The former could arise from the pointing instability of the lasing electron bunches. At hard x-ray wavelengths the pulses from the LCLS should only show a r.m.s. centroid variation which is $10-20 \%$ of the beam size. ${ }^{6}$ The larger angular deviations we measured suggest that either our polystyrene spheres were injected away from the pulse focus where there is substantial phase curvature, or that there were residual phase curvature or phase tilt fluctuations near the pulse focus (within the circular region in Fig. 2).

If the wavefront of the average FEL pulse were convex then Fig. 5 would correspond to the angular map of the average pulse's intensity profile at the interaction region. However, we do not have sufficient evidence to ascertain such convexity. Despite this, the combination of deviations and intensities in Fig. 5 differs considerably from that produced by a spherical Gaussian beam near its focus (Fig. 7). Notice from Fig. 7 that the intensity cross section produced by a spherical Gaussian beam would remain azimuthally symmetric about the beam axis. While similar deviations were previously predicted to arise from the figure and finish of the mirrors used to direct the $\mathrm{x}$-ray pulses into the imaging chamber, ${ }^{14}$ we have insufficient experimental evidence to confirm this. Comparable variations that were observed in the noisy interior of ablation imprints ${ }^{8}$ are similarly inconclusive since they may be an artifact of heating the imprint substrate above its thermal ablation threshold. Nevertheless, similar pulse to pulse intensity fluctuations have been observed with Hartmann sensors at the extreme-ultraviolet FEL at FLASH $^{11}$ which, like LCLS, also generates x-ray lasers via the self-amplified spontaneous emission (SASE) lasing process.

\section{DISCUSSION AND OUTLOOK}

There are obvious limitations to probing FEL pulses with randomly injected polystyrene spheres. Single illuminated spheres only probe the area on each pulse that it intersects. The diameter of the sphere should be much smaller than the pulse's Rayleigh length to minimize the variations in pulse phase tilt and intensity during diffraction. Unlike the fixed lenses on a Hartmann sensor, the unmeasured random positions of our injected spheres forces us to forgo direct spatial information on the pulse's wavefront. An exception is when the pulse's wavefront is convex, allowing us to relate each sphere's transverse position during illumination to a unique translation of its diffraction pattern at the detector. Even then, the sphere's longitudinal position at illumination may still be unknown. Furthermore, it is unclear if the distribution in Fig. 5 applies to every pulse or fluctuates considerably between pulses. Whereas the former only affects randomly injected samples, the latter also impacts fixed target experiments.

Future extensions of this profiling technique should be focused on addressing the various shortcomings and unaccounted errors described in this paper.

\section{ACKNOWLEDGMENTS}

Experiments were carried out at the LCLS, a national user facility operated by Stanford University on behalf of the U.S. Department of Energy (DOE), Office of Basic Energy Sciences. We acknowledge support by the following: Human Frontier Science Program (N.D.L., M.J.B.); AMOS program within the Chemical Sciences, Geosciences, and Biosciences Division of the Office of Basic Energy Sciences, Office of Science, U.S. DOE (N.D.L., R.G.S., C.Y.H., D.S., and M.J.B.); DOE through the SLAC Laboratory Directed Research and Development Program and by Lawrence Livermore National Laboratory under Contract DE-AC52-07NA27344; the Max Planck Society for funding the development and operation of the CAMP instrument within the ASG at CFEL; the Hamburg Ministry of Science and Research and Joachim Herz Stiftung as part of the Hamburg Initiative for Excellence in Research (LEXI); the Hamburg School for Structure and Dynamics in Infection; CBST at UC under Cooperative Agreement No. PHY 0120999. Lawrence Livermore National Laboratory (LLNL) is operated by Lawrence Livermore National Security (LLC) for the U.S. DOE, National Nuclear Security Administration under Contract DE-AC52-07NA27344. Work by LLNL has been supported, in part, by University of California Laboratory Fee grant 09-LR-05-118036-BARA. We thank the staff of the LCLS for their support in carrying out these experiments. We also acknowledge support from the Swedish Research Council, the European Research Council, Knut och Alice Wallenbergs Stiftelse, and the DFG Cluster of Excellence at the Munich Centre for Advanced

Proc. of SPIE Vol. 8504 850403-8 
Photonics. The Max Planck Advanced Study Group at CFEL acknowledges technical support by R. Andritschke, K. Gärtner, O. Hälker, S. Herrmann, A. Hömke, Ch. Kaiser, K.-U. Kühnel, W. Leitenberger, D. Miessner, D. Pietschner, M. Porro, R. Richter, G. Schaller, C. Schmidt, F. Schopper, C.-D. Schröter, Ch. Thamm, A. Walenta, A. Ziegler, and H. Gorke. N. D. Loh would like to thank G.J. Williams for his insightful suggestions and comments.

\section{REFERENCES}

[1] N. D. Loh, M. J. Bogan, V. Elser, A. Barty, S. Boutet, S. Bajt, J. Hajdu, T. Ekeberg, F. R. N. C. Maia, J. Schulz, M. M. Seibert, B. Iwan, N. Tîmneanu, S. Marchesini, I. Schlichting, R. L. Shoeman, L. Lomb, M. Frank, M. Liang, and H. N. Chapman, "Cryptotomography: Reconstructing 3D Fourier Intensities from Randomly Oriented Single-Shot Diffraction Patterns," Physical review letters 104, p. 25501, June 2010.

[2] M. J. Bogan, S. Boutet, H. N. Chapman, S. Marchesini, A. Barty, W. H. Benner, U. Rohner, M. Frank, S. P. Hau-Riege, S. Bajt, B. W. Woods, M. M. Seibert, B. Iwan, N. Timneanu, J. Hajdu, and J. Schulz, "Aerosol Imaging with a Soft X-ray Free Electron Laser," Journal of Aerosol Science and Technology 44(3), pp. 1-6, 2010.

[3] M. M. Seibert, T. Ekeberg, F. R. N. C. Maia, M. Svenda, J. Andreasson, O. Jönsson, D. Odić, B. Iwan, A. Rocker, D. Westphal, M. Hantke, D. P. DePonte, A. Barty, J. Schulz, L. Gumprecht, N. Coppola, A. Aquila, M. Liang, T. A. White, A. Martin, C. Caleman, S. Stern, C. Abergel, V. Seltzer, J.-M. Claverie, C. Bostedt, J. D. Bozek, S. Boutet, A. A. Miahnahri, M. Messerschmidt, J. Krzywinski, G. J. Williams, K. O. Hodgson, M. J. Bogan, C. Y. Hampton, R. G. Sierra, D. Starodub, I. Andersson, S. Bajt, M. Barthelmess, J. C. H. Spence, P. Fromme, U. Weierstall, R. Kirian, M. Hunter, R. B. Doak, S. Marchesini, S. P. HauRiege, M. Frank, R. L. Shoeman, L. Lomb, S. W. Epp, R. Hartmann, D. Rolles, A. Rudenko, C. Schmidt, L. Foucar, N. Kimmel, P. Holl, B. Rudek, B. Erk, A. Hömke, C. Reich, D. Pietschner, G. Weidenspointner, L. Strüder, G. Hauser, H. Gorke, J. Ullrich, I. Schlichting, S. Herrmann, G. Schaller, F. Schopper, H. Soltau, K.-U. Kühnel, R. Andritschke, C.-D. Schröter, F. Krasniqi, M. Bott, S. Schorb, D. Rupp, M. Adolph, T. Gorkhover, H. Hirsemann, G. Potdevin, H. Graafsma, B. Nilsson, H. N. Chapman, and J. Hajdu, "Single mimivirus particles intercepted and imaged with an X-ray laser," Nature 470, pp. 78-81, Feb. 2011.

[4] S. Kassemeyer, J. F. Steinbrener, L. Lomb, E. Hartmann, A. Aquila, A. Barty, A. V. Martin, C. Y. Hampton, S. Bajt, M. Barthelmess, T. R. M. Barends, C. Bostedt, M. Bott, J. D. Bozek, N. Coppola, M. Cryle, D. P. DePonte, R. B. Doak, S. W. Epp, B. Erk, H. Fleckenstein, L. Foucar, H. Graafsma, L. Gumprecht, A. Hartmann, R. Hartmann, G. Hauser, H. Hirsemann, A. Hömke, P. Holl, O. Jönsson, N. Kimmel, F. Krasniqi, M. Liang, F. R. N. C. Maia, S. Marchesini, K. Nass, C. Reich, D. Rolles, B. Rudek, A. Rudenko, C. Schmidt, J. Schulz, R. L. Shoeman, R. G. Sierra, H. Soltau, J. C. H. Spence, D. Starodub, F. Stellato, S. Stern, G. Stier, M. Svenda, G. Weidenspointner, U. Weierstall, T. A. White, C. B. Wunderer, M. Frank, H. N. Chapman, J. Ullrich, L. Strüder, M. J. Bogan, and I. Schlichting, "Femtosecond free-electron laser x-ray diffraction data sets for algorithm development," Optics Express 20(4), pp. 4149-4158, 2012.

[5] N. D. Loh, C. Y. Hampton, A. V. Martin, D. Starodub, R. G. Sierra, A. Barty, A. Aquila, J. Schulz, L. Lomb, J. F. Steinbrener, R. L. Shoeman, S. Kassemeyer, C. Bostedt, J. Bozek, S. W. Epp, B. Erk, R. Hartmann, D. Rolles, A. Rudenko, B. Rudek, L. Foucar, N. Kimmel, G. Weidenspointner, G. Hauser, P. Holl, E. Pedersoli, M. Liang, M. M. Hunter, L. Gumprecht, N. Coppola, C. Wunderer, H. Graafsma, F. R. N. C. Maia, T. Ekeberg, M. Hantke, H. Fleckenstein, H. Hirsemann, K. Nass, T. A. White, H. J. Tobias, G. R. Farquar, W. H. Benner, S. P. Hau-Riege, C. Reich, A. Hartmann, H. Soltau, S. Marchesini, S. Bajt, M. Barthelmess, P. H. Bucksbaum, K. O. Hodgson, L. Strüder, J. Ullrich, M. Frank, I. Schlichting, H. N. Chapman, and M. J. Bogan, "Fractal morphology, imaging and mass spectrometry of single aerosol particles in flight," Nature 486, pp. 513-517, June 2012.

[6] P. Emma, R. Akre, J. Arthur, R. M. Bionta, C. Bostedt, J. Bozek, A. Brachmann, P. H. Bucksbaum, R. Coffee, F. J. Decker, Y. Ding, D. Dowell, S. Edstrom, A. Fisher, J. Frisch, S. Gilevich, J. Hastings, G. Hays, P. Hering, Z. Huang, R. Iverson, H. Loos, M. Messerschmidt, A. Miahnahri, S. Moeller, H.-D. Nuhn, D. Pile, D. Ratner, J. Rzepiela, D. Schultz, T. Smith, P. Stefan, H. Tompkins, J. Turner, J. Welch, W. White, J. Wu, G. Yocky, and J. N. Galayda, "First lasing and operation of an angstrom-wavelength free-electron laser," Nature Photonics 4, pp. 641-647, 2010. 
[7] J. Chalupsky, L. Juha, J. Kuba, J. Cihelka, V. Hajkova, S. Koptyaev, J. Krása, A. Velyhan, M. Bergh, C. Caleman, J. Hajdu, R. M. Bionta, H. N. Chapman, S. P. Hau-Riege, R. A. London, M. Jurek, J. Krzywinski, R. Nietubyc, J. B. Pelka, R. Sobierajski, J. Meyer-ter Vehn, A. Tronnier, K. Sokolowski-Tinten, N. Stojanovic, K. Tiedtke, S. Toleikis, T. Tschentscher, H. Wabnitz, and U. Zastrau, "Characteristics of focused soft X-ray free-electron laser beam determined by ablation of organic molecular solids," Optics Express 15, pp. 6036-6043, May 2007.

[8] J. Chalupsky, P. Bohacek, V. Hajkova, S. P. Hau-Riege, P. A. Heimann, L. Juha, J. Krzywinski, M. Messerschmidt, S. P. Moeller, B. Nagler, M. Rowen, W. F. Schlotter, M. L. Swiggers, and J. J. Turner, "Comparing different approaches to characterization of focused X-ray laser beams," Nuclear Instruments and Methods in Physics Research A 631, pp. 130-133, 2011.

[9] S. P. Hau-Riege, H. N. Chapman, J. Krzywinski, R. Sobierajski, S. Bajt, R. A. London, M. Bergh, C. Caleman, R. Nietubyc, and L. Juha, "Subnanometer-scale measurements of the interaction of ultrafast soft X-ray free-electron-laser pulses with matter," Physical review letters 98(14), p. 145502, 2007.

[10] S. Le Pape, P. Zeitoun, M. Idir, P. Dhez, J. Rocca, and M. François, "Electromagnetic-Field Distribution Measurements in the Soft X-Ray Range: Full Characterization of a Soft X-Ray Laser Beam," Physical review letters 88, p. 183901, Apr. 2002.

[11] B. Flöter, P. Juranić, S. Kapitzki, B. Keitel, K. Mann, E. Plönjes, B. Schafer, and K. Tiedtke, "EUV Hartmann sensor for wavefront measurements at the Free-electron LASer in Hamburg," New Journal of Physics 12, p. 083015, 2010.

[12] M. Idir, P. Mercère, M. Modi, G. Dovillaire, X. Levecq, S. Bucourt, L. Escolano, and P. Sauvageot, "X-ray active mirror coupled with a Hartmann wavefront sensor," Nuclear Inst and Methods in Physics Research A 616, pp. 162-171, 2010.

[13] P. Dorenbos, J. de Haas, and C. Van Eijk, "Non-proportionality in the scintillation response and the energy resolution obtainable with scintillation crystals," Nuclear Science, IEEE Transactions on 42(6), pp. 21902202, 1995.

[14] A. Barty, R. Soufli, T. McCarville, S. L. Baker, M. J. Pivovaroff, P. Stefan, and R. M. Bionta, "Predicting the coherent X-ray wavefront focal properties at the Linac Coherent Light Source (LCLS) X-ray free electron laser," Optics Express 17(18), pp. 15508-15519, 2009.

[15] J. D. Bozek, "AMO instrumentation for the LCLS X-ray FEL," The European Physical Journal-Special Topics 169, pp. 129-132, 2009.

[16] W. H. Benner, M. J. Bogan, U. Rohner, S. Boutet, B. W. Woods, and M. Frank, "Non-destructive characterization and alignment of aerodynamically focused particle beams using single particle charge detection," Journal of Aerosol Science and Technology 39, pp. 917-928, 2008.

[17] M. J. Bogan, W. H. Benner, S. Boutet, U. Rohner, M. Frank, A. Barty, M. M. Seibert, F. R. N. C. Maia, S. Marchesini, S. Bajt, B. W. Woods, V. Riot, S. P. Hau-Riege, M. Svenda, E. Marklund, E. Spiller, J. Hajdu, and H. N. Chapman, "Single Particle X-ray Diffractive Imaging," Nano Letters 8(1), pp. 310-316, 2008.

[18] L. Strüder, S. Epp, D. Rolles, R. Hartmann, et al., "Large-format, high-speed, X-ray pnCCDs combined with electron and ion imaging spectrometers in a multipurpose chamber for experiments at 4th generation light sources," Nuclear Instruments and Methods in Physics Research A 614, pp. 483-496, 2010. 